\title{
Prescriptions patterns and appropriateness of usage of antibiotics in non-teaching community hospitals in South Korea: a multicentre retrospective study
}

Yong Chan Kim ${ }^{1+}$, Ji Young Park ${ }^{2 \dagger}$, Bongyoung Kim ${ }^{3^{*}+}$, Eu Suk Kim ${ }^{4^{*}+}$, Hyuk Ga ${ }^{5}$, Rangmi Myung ${ }^{6}$, Se Yoon Park , Myung Jin Lee ${ }^{8}$, Song Mi Moon ${ }^{9}$, Sun Hee Park ${ }^{10}$, Kyoung-Ho Song ${ }^{4}$, Hong Bin Kim ${ }^{4}$ and Korea Study Group for Antimicrobial Stewardship (KOSGAP)

\begin{abstract}
Background: Although non-teaching community hospitals form the majority of healthcare providers in South Korea, there is limited data on antibiotic usage in them. To evaluate the pattern of antibiotic usage and its appropriateness in hospitals with $<400$ beds in South Korea.

Methods: A multicentre retrospective study was conducted in 10 hospitals (six long-term care hospitals, three acute care hospitals, and one orthopaedic hospital), with $<400$ beds in South Korea. We analysed patterns of antibiotic prescription in 2019, and their appropriateness in the participating hospitals. For the evaluation of the appropriateness of the prescription, 25 patients under antibiotic therapy were randomly selected at each hospital, over two separate periods. Due to the heterogeneity of their characteristics, the orthopaedics hospital was excluded from the analysis.

Results: The most commonly prescribed antibiotics in long-term care hospitals was fluoroquinolone, followed by beta-lactam/beta-lactamase inhibitor (anti-pseudomonal). In acute care hospitals, these were third generation cephalosporin, followed by first generation cephalosporin, and second generation cephalosporin. The major antibiotics that were prescribed in the orthopedics hospital was first generation cephalosporin Only $2.3 \%$ of the antibiotics were administered inappropriately. In comparison, $15.3 \%$ of patients were prescribed an inappropriate dose. The proportion of inappropriate antibiotic prescriptions was $30.6 \%$ of the total antibiotic prescriptions.
\end{abstract}

Conclusions: The antibiotic usage patterns vary between non-teaching community hospitals in South Korea. The proportion of inappropriate prescriptions exceeded 30\% of the total antibiotic prescriptions.

\footnotetext{
*Correspondence: sobakas@hanyang.ac.kr; eskim@snubh.org

†Yong Chan Kim and Ji Young Park contributed equally as first authors

${ }^{\dagger}$ Bongyoung Kim and Eu Suk Kim contributed equally as corresponding

authors

${ }^{3}$ Department of Internal Medicine, Hanyang University College

of Medicine, 222-1, Wangsimni-ro, Seongdong-gu, Seoul 04763, Korea

${ }^{4}$ Division of Infectious Diseases, Department of Internal Medicine, Seoul

National University Bundang Hospital, Seoul National University College

of Medicine, 82 Gumi-ro, 173 beon-gil, Bundang-gu, Seongnam 13620,

Gyeonggi-do, Korea

Full list of author information is available at the end of the article
}

(c) The Author(s) 2022. Open Access This article is licensed under a Creative Commons Attribution 4.0 International License, which permits use, sharing, adaptation, distribution and reproduction in any medium or format, as long as you give appropriate credit to the original author(s) and the source, provide a link to the Creative Commons licence, and indicate if changes were made. The images or other third party material in this article are included in the article's Creative Commons licence, unless indicated otherwise in a credit line to the material. If material is not included in the article's Creative Commons licence and your intended use is not permitted by statutory regulation or exceeds the permitted use, you will need to obtain permission directly from the copyright holder. To view a copy of this licence, visit http://creativecommons.org/licenses/by/4.0/. The Creative Commons Public Domain Dedication waiver (http://creativeco mmons.org/publicdomain/zero/1.0/) applies to the data made available in this article, unless otherwise stated in a credit line to the data. 
Keywords: Antibiotics, Stewardship, Resistance, Infectious diseases, Korea

\section{Introduction}

Antibiotics are one of the most significant discoveries in modern medical history. These drugs have saved innumerable lives; the timely administration of appropriate antibiotics, in particular, can reduce morbidity and mortality in patients with infectious diseases $[1,2]$. Despite their obvious benefits, however, the misuse of antibiotics can lead to an increase in antibiotic-resistant pathogens as well as infectious diseases caused by these pathogens $[3,4]$. Additionally, excessive use of antibiotics is related to Clostridioides difficile infections or adverse drug events, which may require additional hospital stay, consequently increasing healthcare costs $[5,6]$.

To reduce such harmful effects, optimize antibiotic use for infectious diseases, and minimize unnecessary use, the Antimicrobial Stewardship Program (ASP) was implemented globally [7]. The first step in implementing effective ASPs and assessing effective interventions is to understand the patterns of antibiotic use in hospitals [8, 9]. Therefore, the measurement of antibiotic prescriptions and the evaluation of antibiotic use form one of the core elements of ASP [7].

Most studies on antibiotic use and appropriateness of prescriptions in South Korea have been performed in large academic centres [10, 11]. Unfortunately, even though determining antibiotic use and prescription patterns is included in the Korean National Action Plan on antimicrobial resistance 2016-2020, there is limited data on the patterns of antibiotic use in non-teaching community hospitals [12]. Indeed, non-teaching community hospitals form the majority of healthcare providers in South Korea; in 2018, 94.5\% of all hospitals had $<400$ beds, with a mean bed size of 145 [13]. Therefore, we performed a study to evaluate the pattern of antibiotic usage and its appropriateness in non-teaching hospitals with $<400$ beds in South Korea.

\section{Material and methods Study settings}

To recruit hospitals, an e-mail containing information about the research was sent to non-teaching hospitals with $<400$ beds via Infection Control Consulting Network in South Korea. Ten hospitals submitted applications for participation, and were included in our multicentre retrospective study. Of the participating hospitals, 6 (A-F) were long-term care hospitals, 3 (G-I) were acute care (general) hospitals, and 1 (J) was an orthopaedic hospital. The number of hospital beds ranged from 99 to 361 , and $60 \%$ of the participating hospitals were in metropolitan areas (Seoul, Incheon, and Gyeonggi-do). The hospital names have been blinded to safeguard them from the possibility of unintended blame. The overall characteristics of the participating hospitals are listed in Table 1.

We analysed the antibiotic prescription patterns and their appropriateness in the participating hospitals. However, due to the heterogeneity of patient characteristics and antibiotic prescription patterns, hospital J (orthopaedics hospital) was excluded from the evaluation of the appropriateness of antibiotic prescription.

In this study, antibiotics were defined as medication with Anatomical Therapeutic Chemical class J01, which does not include antifungal, antituberculotic, antiparasitic, or antiviral agents. Systemic agents with oral or parenteral administration routes were included, whereas topical agents were excluded.

The study's protocol was approved by the Institutional Review Board of Hanyang University, Seoul Hospital (IRB no. 2020-12-040). The requirement for an informed written consent from patients was waived because of the retrospective nature of the study.

\section{Analysis of antibiotic prescription patterns}

Data on the monthly antibiotic prescriptions and patient-days for hospitalized patients were collected using electronic databases from each hospital. To avoid the effect of the coronavirus pandemic, data were collected from January to December 2019. The amount of antibiotic consumption was calculated using the defined daily dose (DDD) and days of therapy (DOT), and then standardized for 1000 patient-days [14]. We categorized antibiotic agents into 19 classes [10]: first generation cephalosporin (1G CEP), second generation cephalosporin (2G CEP), third generation cephalosporin (3G CEP), fourth generation cephalosporin, aminoglycoside, $\beta$-lactam/ $\beta$-lactamase inhibitor (BL/BLI), BL/BLI (anti-pseudomonal), carbapenem, fluoroquinolone (FQ), glycopeptide, lincosamide, macrolide, metronidazole, oxazolidinone, penicillin, polymyxin, sulfonamide and trimethoprim, tetracycline, and tigecycline. Other antibiotics were excluded because they were rarely used.

\section{Evaluating the appropriateness of antibiotic prescription}

To assess the appropriateness of antibiotic prescriptions, investigators in each hospital retrospectively collected information on patients with such prescriptions, according to the data collection forms. The collected data included patient information, information on antibiotic 


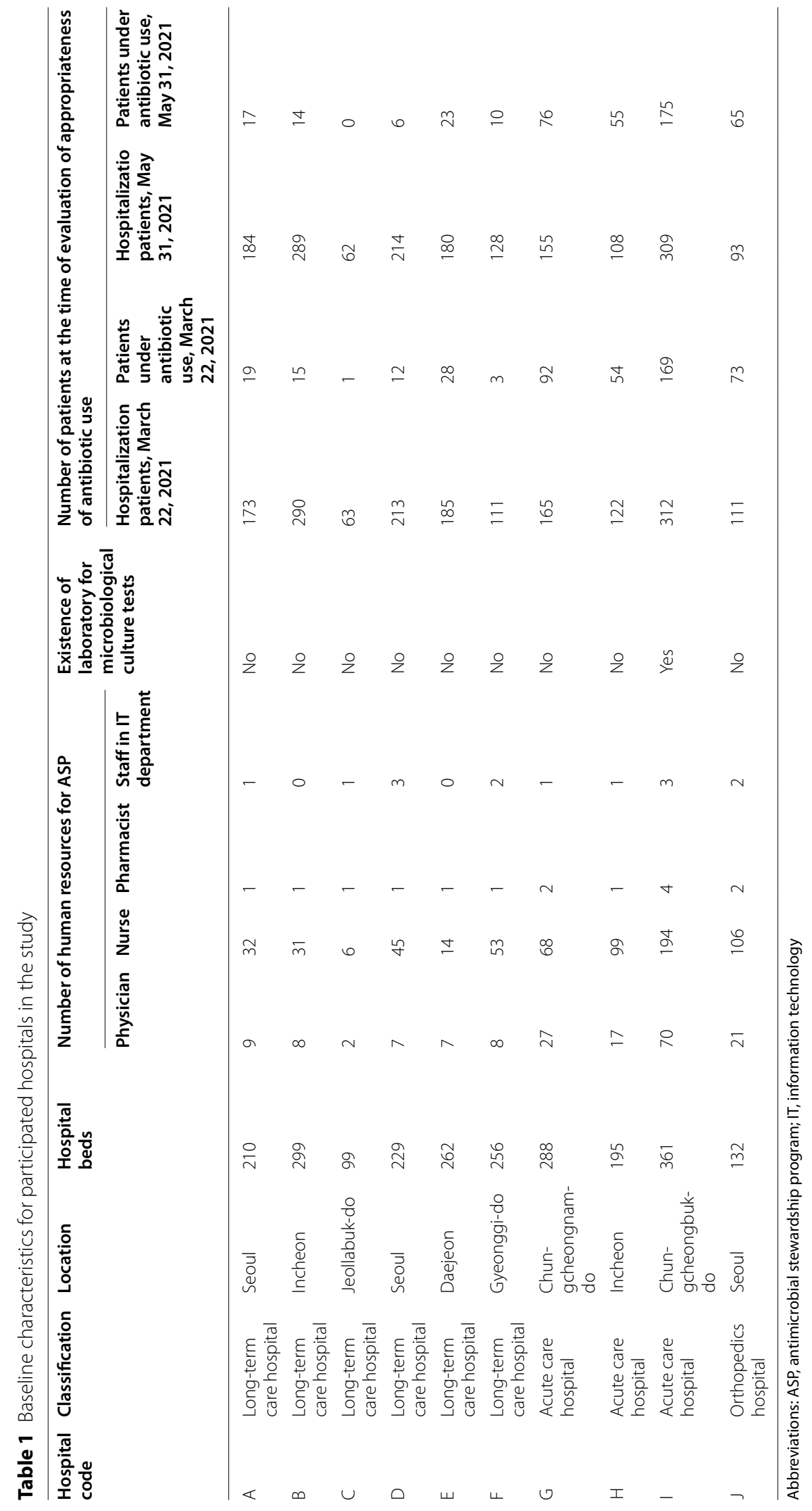


prescription, test results from microbiological cultures, and clinical information related to infectious diseases (Additional file 1: Supplement 1). The data were collected from two separate periods (22nd March 2021-21st April 2021 and 31st May 2021-30th June 2021) to minimise the effect of temporal bias. Investigators in each hospital collected cases that they treated in orders of the latest date, and then the assessment targets were randomly selected-a total of 25 patients in each hospital for each period; if the number of patients was less than 25 , all patients were included. The assessment targets required that one patient be included only once; further, new-born babies ( $<28$ days old) were excluded.

The collected data were reviewed and the appropriateness of antibiotic prescriptions was evaluated by five specialists in infectious diseases (adult and paediatric). Data from two hospitals were assigned to each specialist. The appropriateness of antibiotic prescriptions was evaluated from three aspects: route of administration, dose, and class [15]. If the three aspects were 'optimal', the prescription was considered 'optimal'; if only the route was 'optimal', and the dose and/or class was 'suboptimal' but not 'inappropriate', it was considered 'suboptimal'; if even one aspect was 'inappropriate', it was classified as 'inappropriate'.

Further, we assessed the appropriateness of the prescription by the patient: if all the prescribed antibiotics were evaluated as 'optimal' and there was no unnecessary combination, the antibiotic prescription for the patient was considered 'optimal'; in case of at least one 'suboptimal' antibiotic prescription, but no 'inappropriate' antibiotic prescription, it was considered 'suboptimal.' However, even if all antibiotic prescriptions were 'optimal' but the combination was unnecessary, it was considered 'suboptimal'. If even one 'inappropriate' antibiotic prescription existed, it was considered as such (Table 2 and Additional file 2: Supplement 2).

We evaluated the appropriateness of the route of administration and dose using the Sanford guide to antimicrobial therapy [16]. Antibiotics administered via a route recommended in the Sanford guide were considered to have an 'appropriate' route of administration; the rest were considered 'inappropriate'. The appropriateness of the dose was evaluated by analysing the patients' renal function. Hence, the evaluation of antibiotics that needed a renal dose adjustment was conducted only when the results of the renal function test existed; cases without a result were considered 'N/A'. If the dose was within the range of recommendation of the Sanford guide, it was considered 'optimal'; if the dose was significantly higher than the recommended amount, it was considered 'suboptimal', and if the dose was significantly lower than the recommended amount, it was considered 'inappropriate'.
The standards for the evaluation of the appropriateness of antibiotic classes differed according to the objectives of the prescription. If the antibiotic was prescribed for the treatment of an infectious disease, the appropriateness of the antibiotic class was assessed in accordance with antibiograms of isolated pathogens, clinical practice guidelines for infectious diseases, and the Sanford guide to antimicrobial therapy (Additional file 3: Supplement 3). Given that the antibiotic prescription might be evaluated as 'inappropriate' when the diagnosis of infectious diseases was incorrect, we also evaluated the appropriateness of the diagnosis; the standard for the evaluation of each infectious disease was distributed to infectious disease specialists (Additional file 3: Supplement 4). Prescribed antibiotics recommended in the guidelines and antibiotics susceptible to identified or possible pathogen were considered 'optimal'; if the beta-lactam or antistaphylococcal antibiotic spectrum was too broad considering identified or possible pathogen, it was evaluated as 'suboptimal'; the rest were considered 'inappropriate'. If the antibiotic was prescribed for prophylaxis in the prevention of surgical site infection, the appropriateness of antibiotic class was assessed using the criteria of 'The ninth nationwide evaluation of the appropriateness of surgical prophylactic antibiotics in Korean hospitals in 2020', led by the Health Insurance Review and Assessment Service [17]; if the prescribed antibiotics adhered to the criteria, they were considered 'appropriate'; the rest were considered 'inappropriate'. If the objective of the prescription did not belong to the treatment of infectious disease or prophylaxis for prevention of surgical site infection, the appropriateness was decided by the judgment of the designated infectious disease specialist (Table 2 and Additional file 2: Supplement 2).

\section{Statistical analysis}

The results from evaluating the appropriateness of antibiotic prescriptions compared the differences between long-term care hospitals and acute care hospitals. All statistical analyses were conducted using SPSS version 24.0 for Windows (IBM Corp., Armonk, NY, USA). The categorical variables were analysed using the chi-square test or Fisher's exact test, and the continuous variables were analysed using the Mann-Whitney $U$ test. A two-tailed $P$-value of $<0.05$ was considered statistically significant, for this study's parameters.

\section{Results}

\section{Antibiotic prescription patterns}

The antibiotics being used in each hospital are described in Additional file 3: Supplement 5. The number of antibiotics used in each hospital ranged from 4 to 15 in long-term care hospitals, and from 24 to 36 in acute care 


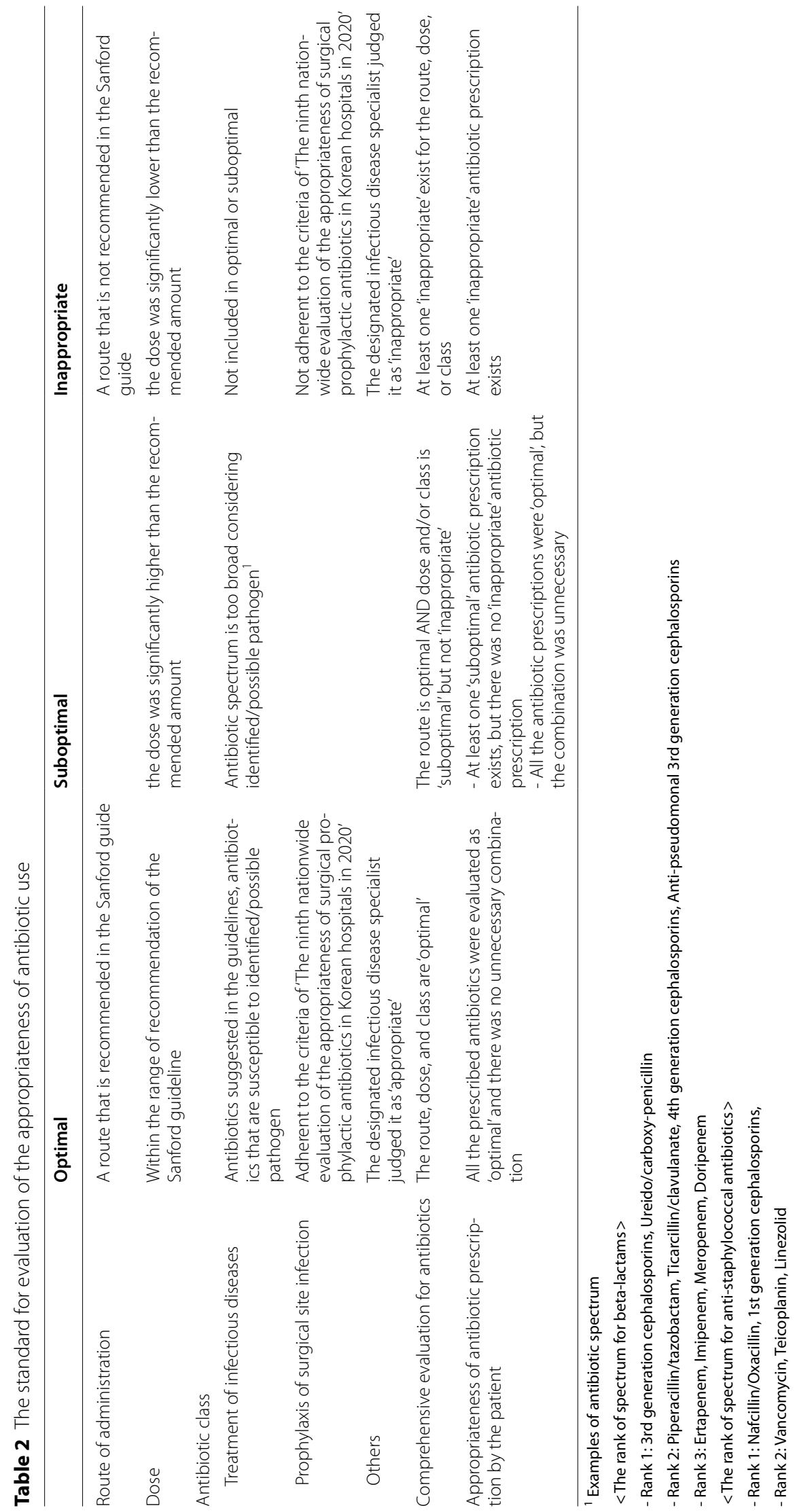


hospitals. Twelve antibiotics were used in the orthopaedic hospital (hospital J).

The total amount of antibiotic consumption in 2019 ranged from 29.9 to $168.6 \mathrm{DDD} / 1000$ patient-days (60.1116.1 DOT/1000 patient-days) in long-term care hospitals, and from 546.7 to $674.9 \mathrm{DDD} / 1000$ patient-days (577.5-616.6 DOT/1000 patient-days) in acute care hospitals. In the orthopaedic hospital, the total amount of antibiotic consumption was $410.1 \mathrm{DDD} / 1000$ patientdays (563.9 DOT/1000 patient-days) (Additional file 3: Supplement 6).

The most commonly prescribed antibiotic in long-term care hospitals was FQ (23.1\% by DDD; $20.9 \%$ by DOT), followed by BL/BLI (anti-pseudomonal) (10.9\% by DDD; $9.9 \%$ by DOT). In acute care hospitals, it was $3 \mathrm{G} \mathrm{CEP}$ (19.9\% by DDD; $21.3 \%$ by DOT), followed by $1 \mathrm{G}$ CEP ( $16.9 \%$ by DDD; $20.8 \%$ by DOT) and $2 \mathrm{G} \mathrm{CEP}(15.3 \%$ by DDD; $17.7 \%$ by DOT). The proportion of carbapenem use in long-term and acute care hospitals was $5.2 \%$ by DDD $(6.6 \%$ by DOT) and $11.6 \%$ by DDD ( $9.4 \%$ by DOT), respectively. The major antibiotics prescribed in the orthopaedic hospital was 1G CEP (91.3\% by DDD; $92.4 \%$ by DOT) (Fig. 1). There were some differences in the frequency of antibiotics used among individual institutions, even within the same type of hospitals (Fig. 2).

\section{Appropriateness of antibiotic prescriptions}

A total of 422 patients (272 from long-term and 150 from acute care hospitals) were recruited to study the appropriateness of antibiotic usage. A total of 569 antibiotics (384 from long-term and 185 from acute care hospitals) that were prescribed to these patients were evaluated.

Table 3 and Additional file 3: Supplement 7 show the baseline characteristics of the patients. The median age of patients from long-term care hospitals was 80.0 and higher than those from acute care hospitals (median 73.0) $(P<0.001)$. The proportion of females in long-term and acute care hospitals was $50.7 \%$ and $52.0 \%$, respectively $(P=0.804)$. Data on renal function, which included the estimated glomerular filtration ratio, was available from the electronic medical record for $27.9 \%$ of the patients from long-term care hospitals, and $76.0 \%$ of the patients from acute care hospitals $(P<0.001)$. The proportion of patients with cognitive disorders $(88.1 \%$ vs. $48.0 \%, P<0.001)$ and in bedridden state $(62.9 \%$ vs. $77.0 \%$, $P<0.001)$ were higher in long-term care hospitals than in acute care hospitals. The proportion of patients who had results of microbiological culture with blood samples $(12.1 \%$ vs. $68.5 \%, P<0.001)$ and non-blood samples $(13.6 \%$ vs. $69.6 \%, P<0.001)$ were higher in acute care hospitals than in long-term care hospitals.

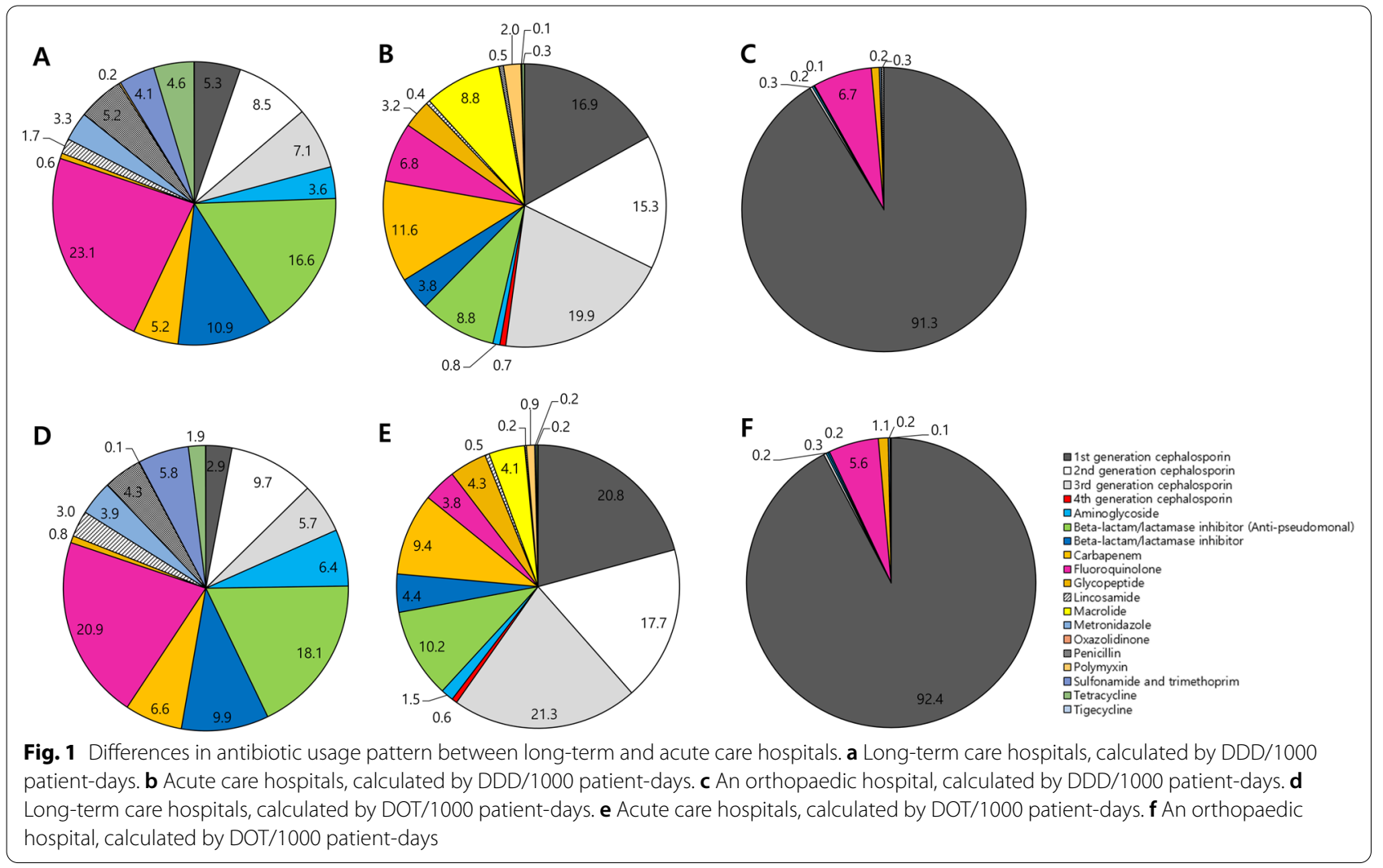



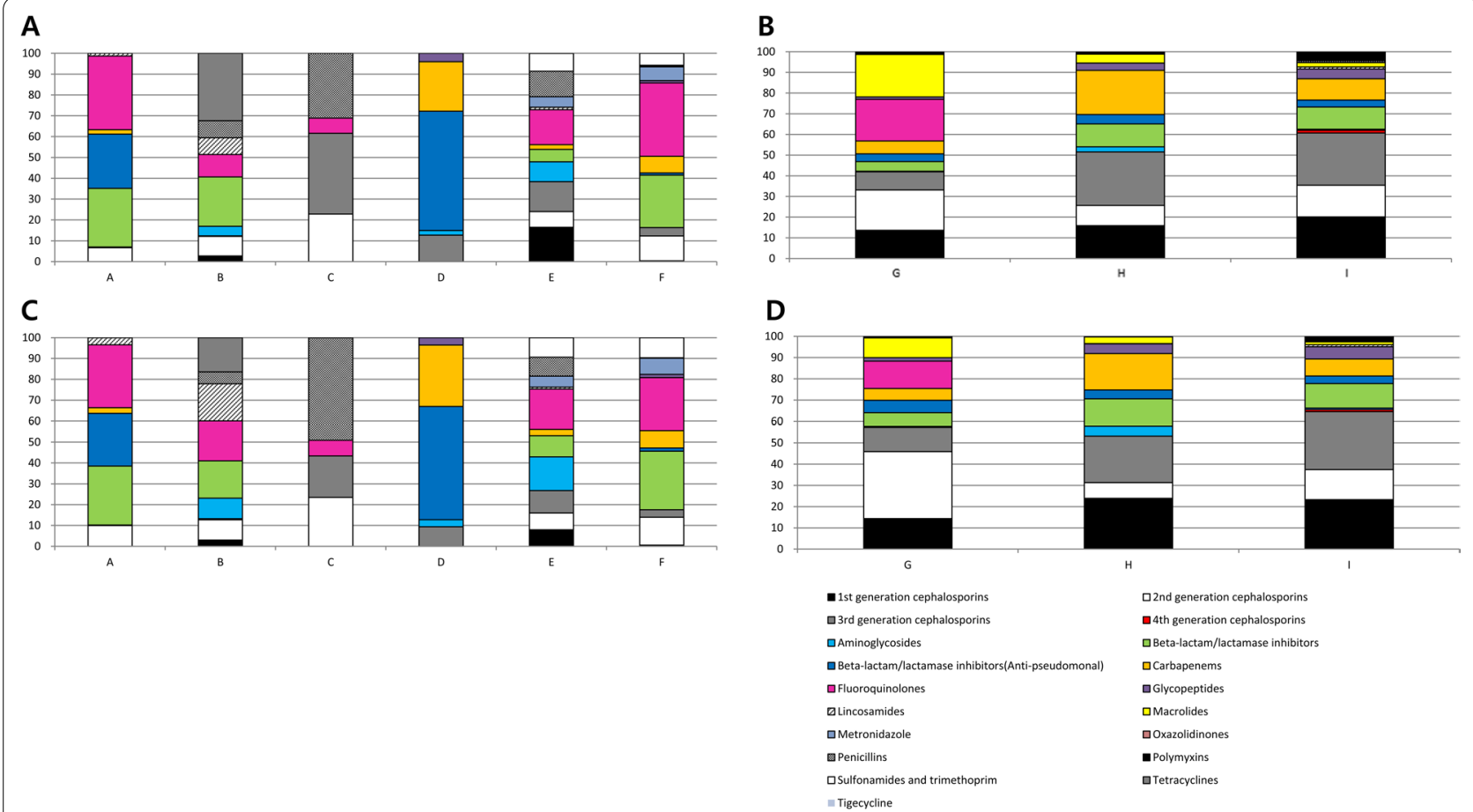

Fig. 2 Difference in antibiotic usage pattern among hospitals. a Long-term care hospitals, calculated by DDD/1000 patient-days. b Acute care hospitals, calculated by DDD/1000 patient-days. c Long-term care hospitals, calculated by DOT/1000 patient-days. $\mathbf{d}$ Acute care hospitals, calculated by DOT/1000 patient-days

Table 3 Baseline characteristics for patients in the study for evaluation of appropriateness of antibiotic use

\begin{tabular}{|c|c|c|c|c|}
\hline & $\begin{array}{l}\text { Long-term care hospitals } \\
(\mathrm{N}=272)\end{array}$ & $\begin{array}{l}\text { Acute care hospitals } \\
(\mathrm{N}=150)\end{array}$ & $P$-value & $\begin{array}{l}\text { All hospitals } \\
(\mathrm{N}=422)\end{array}$ \\
\hline Age, median (IQR) & $80.0(72.0-86.0)$ & $73.0(61.0-82.0)$ & $<0.001$ & $79.0(67.0-85.0)$ \\
\hline Female sex (\%) & $138(50.7)$ & $78(52.0)$ & 0.804 & $216(51.2)$ \\
\hline \multicolumn{5}{|l|}{ Ward type (\%) } \\
\hline General ward & $260(95.6)$ & $118(78.7)$ & $<0.001$ & $378(89.6)$ \\
\hline Intensive care unit & $12(4.4)$ & $32(21.3)$ & - & $44(10.4)$ \\
\hline \multicolumn{5}{|l|}{ Classification of department (\%) } \\
\hline Internal Medicine & $68 / 270(25.2)$ & $97 / 149(65.1)$ & $<0.001$ & $165 / 419(39.4)$ \\
\hline Medical department (excluding internal medicine) & $162 / 270(60.0)$ & $5 / 149(3.4)$ & - & $167 / 419(39.9)$ \\
\hline Surgical department & $40 / 270(14.8)$ & $47 / 149(31.5)$ & - & $87 / 419(20.8)$ \\
\hline \multicolumn{5}{|l|}{ Data about renal function at EMR (\%) } \\
\hline Existence of result of $\mathrm{CrCl}$ & $72(26.5)$ & $50(33.3)$ & 0.137 & $122(28.9)$ \\
\hline Existence of result of eGFR & $76(27.9)$ & $114(76.0)$ & $<0.001$ & $190(45.0)$ \\
\hline Patients underwent renal replacement therapy (\%) & $24 / 270(8.9)$ & $6(4.0)$ & 0.062 & $30 / 420(7.1)$ \\
\hline Patients with cognitive disorder (\%) & $238 / 270(88.1)$ & $72(48.0)$ & $<0.001$ & $310 / 420(73.8)$ \\
\hline \multicolumn{5}{|l|}{ Ambulation status } \\
\hline Ambulation, regardless of external support & $17 / 270(6.3)$ & $69(68.5)$ & $<0.001$ & $86 / 420(20.5)$ \\
\hline Ambulation with wheelchair & $45 / 270(16.7)$ & $25(16.7)$ & - & $70 / 420(16.7)$ \\
\hline Bed-ridden status & 208/270 (77.0) & $56(37.3)$ & - & $264 / 420(62.9)$ \\
\hline \multicolumn{5}{|l|}{ Microbiological culture test } \\
\hline Existence of result of culture with blood sample & $33(12.1)$ & $102 / 149(68.5)$ & $<0.001$ & 135/421 (32.1) \\
\hline Existence of result of culture with non-blood sample & $37(13.6)$ & 103/148 (69.6) & $<0.001$ & $140 / 420(33.3)$ \\
\hline
\end{tabular}

IQR interquartile range, EMR electronic medical record, $\mathrm{CrCl}$ creatinine clearance, eGFR estimated glomerular filtrationrate 
Figure 3, and Additional file 3: Supplements 8 and 9 show the characteristics of antibiotics evaluated in this study. The proportion of parenteral antibiotics were $63.8 \%$ in long-term care hospitals and $97.8 \%$ in acute care hospitals $(P<0.001)$. The majority of the evaluated antibiotics were prescribed to treat infectious diseases in both types of hospitals $(96.3 \%$ vs. $83.8 \%, P<0.001)$. The most common infectious disease was respiratory tract infections $(48.5 \%)$, followed by genitourinary tract infections (23.0\%), and gastrointestinal tract infections (9.2\%).

Table 4 and Additional file 3: Supplement 10 show the appropriateness of the antibiotic prescriptions. Only $2.3 \%$ of antibiotics were administered inappropriately; the proportion of antibiotics with inappropriate routes of administration was not very different between longterm and acute care hospitals ( $2.6 \%$ vs. $1.6 \%, P=0.562)$. In comparison, $15.3 \%$ patients were prescribed an inappropriate dose; the proportion of antibiotics with inappropriate doses were higher in long-term care hospitals than in acute care hospitals $(16.1 \%$ vs. $13.5 \%, P<0.001)$. As for the choice of antibiotics, $33.0 \%$ were evaluated as being inappropriate for treating infectious disease, and $45.5 \%$ were evaluated as inappropriate for the prophylaxis of surgical site infections. The proportion of inappropriate antibiotic choice for the treatment of infectious disease was higher in long-term care hospitals than in acute care hospitals $(34.9 \%$ vs. $28.4 \%$, $P=0.034)$. The proportion of inappropriate antibiotic prescriptions was $30.6 \%$ of the total antibiotic prescriptions, for the route, dose, and class of administration; $40.2 \%$ could not be evaluated due to insufficient data. In comparison, the proportion of patients with inappropriate antibiotic prescriptions was $32.0 \%$ of all patients who were prescribed antibiotics, and $38.2 \%$ could not be evaluated. The proportion of inappropriate antibiotic prescriptions $(28.4 \%$ vs. $35.1 \%, P<0.001)$ and that of patients with inappropriate antibiotics $(31.3 \%$ vs. $33.3 \%, P<0.001)$ were higher in acute care hospitals than in long-term care hospitals. The proportion of antibiotics that could not be evaluated $(51.3 \%$ vs. $17.3 \%$, $P<0.001)$ and those prescribed with antibiotics $(48.2 \%$ vs. $20.0 \%, P<0.001)$ were higher in long-term care hospitals.

When cases with an inappropriate diagnosis were excluded, the proportion of inappropriate antibiotic prescriptions decreased to $21.2 \%$, and that of patients with inappropriate antibiotic prescriptions decreased to $23.2 \%$ (Table 5). FQ (28.7\%) and 3G CEP (19.5\%) constituted the largest proportion of inappropriate antibiotic prescriptions (Additional file 3: Supplement 11). Of the common infectious diseases, the proportion of inappropriate antibiotic prescriptions was higher for respiratory tract infections $(41.8 \%)$ than for genitourinary tract infections (39.2\%) (Additional file 3: Supplement 12).
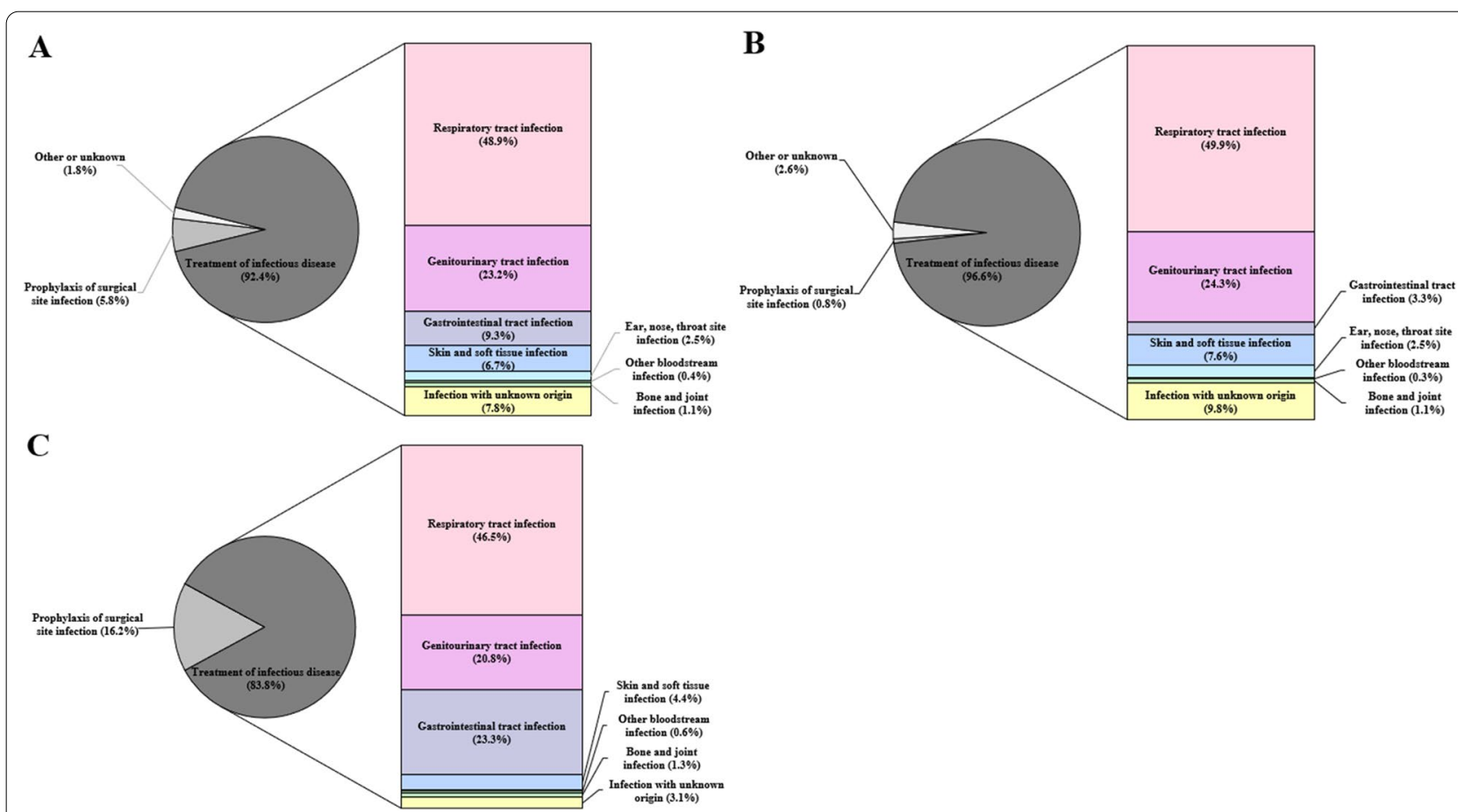

Fig. 3 Purpose of antibiotic prescription. a All hospitals. b Long-term care hospitals. c Acute care hospitals 
Table 4 Appropriateness of antibiotic prescriptions

\begin{tabular}{|c|c|c|c|c|}
\hline & $\begin{array}{l}\text { Long-term care hospitals } \\
(\mathrm{N}=384)\end{array}$ & $\begin{array}{l}\text { Acute care hospitals } \\
(\mathrm{N}=185)\end{array}$ & $P$-value & $\begin{array}{l}\text { All hospitals } \\
(\mathrm{N}=569)\end{array}$ \\
\hline Route of administration (\%) & & & 0.562 & \\
\hline Appropriate & $374(97.4)$ & $182(98.4)$ & & $556(97.7)$ \\
\hline Inappropriate & $10(2.6)$ & $3(1.6)$ & & $13(2.3)$ \\
\hline Dose (\%) & & & $<0.001$ & \\
\hline Optimal & $112(29.2)$ & $139(75.1)$ & & $251(44.1)$ \\
\hline Suboptimal: excessively high dose & $32(8.3)$ & $2(1.1)$ & & $34(6.0)$ \\
\hline Inappropriate: excessively low dose & $62(16.1)$ & $25(13.5)$ & & $87(15.3)$ \\
\hline N/A & $178(46.4)$ & $19(10.3)$ & & $197(34.6)$ \\
\hline \multicolumn{5}{|l|}{ Antibiotic choice (\%) } \\
\hline Antibiotics for the treatment of infectious diseases & & & 0.034 & \\
\hline Optimal & $146 / 370(39.5)^{1}$ & $82 / 155(52.9)$ & & $228 / 525(43.4)^{1}$ \\
\hline Suboptimal & $60 / 370(16.2)^{1}$ & $16 / 155(10.3)$ & & $76 / 525(14.5)^{1}$ \\
\hline Inappropriate & $129 / 370(34.9)^{1}$ & $44 / 155(28.4)$ & & $173 / 525(33.0)^{1}$ \\
\hline N/A & $35 / 370(9.5)^{1}$ & 13/155 (8.4) & & $48 / 525(9.1)^{1}$ \\
\hline Antibiotics for the prophylaxis of surgical site infection & & & 0.579 & \\
\hline Appropriate & $1 / 3(33.3)$ & $17 / 30(56.7)$ & & $18 / 33(54.5)$ \\
\hline Inappropriate & $2 / 3(66.7)$ & $13 / 30(43.3)$ & & $15 / 33(45.5)$ \\
\hline Antibiotics for other or unknown reasons & & & - & \\
\hline Appropriate & 0 & 0 & & 0 \\
\hline Inappropriate & $10 / 10(100)$ & $10 / 10(100)$ & & $10 / 10(100)$ \\
\hline Appropriateness of antibiotic prescription, by each antibiotic (\%) & & & $<0.001$ & \\
\hline Optimal & $49 / 384(12.8)$ & $76 / 185(41.1)$ & & $125 / 569(22.0)$ \\
\hline Suboptimal & 29/384 (7.6) & $12 / 185(6.5)$ & & $41 / 569(7.2)$ \\
\hline Inappropriate & 109/384 (28.4) & $65 / 185(35.1)$ & & $174 / 569(30.6)$ \\
\hline N/A & 197/384 (51.3) & $32 / 185(17.3)$ & & $229 / 569(40.2)$ \\
\hline Appropriateness of antibiotic prescription, by each patient (\%) & & & $<0.001$ & \\
\hline Optimal & $29 / 272(10.7)$ & $57 / 150(38.0)$ & & $86 / 422(20.4)$ \\
\hline Suboptimal: one or more antibiotics were suboptimal & 26/272 (9.6) & $9 / 150(6.0)$ & & $35 / 422(8.3)$ \\
\hline Suboptimal: unnecessary combination therapy & $1 / 272(0.4)$ & $4 / 150(2.7)$ & & $5 / 422(1.2)$ \\
\hline Inappropriate & $85 / 272(31.3)$ & $50 / 150(33.3)$ & & $135 / 422(32.0)$ \\
\hline N/A & $131 / 272(48.2)$ & $30 / 150(20.0)$ & & $161 / 422(38.2)$ \\
\hline
\end{tabular}

${ }^{1}$ One case was excluded because the data was insufficient

\section{Discussion}

This study is the first to analyse the appropriateness of antibiotic usage patterns in non-teaching community hospitals in South Korea. Interestingly, antibiotic use was highly varied among individual hospitals. We found that hospital size was not a predictor of antibiotic use, consistent with previous studies $[18,19]$.

The antibiotic prescription pattern of acute care hospitals was similar to that of large academic hospitals in South Korea [10, 20]. The antibiotic prescription pattern of long-term care hospitals in South Korea has not been evaluated, and we found that the pattern was diverse varying across hospitals. Despite this, only three classes of antibiotics constituted more than half of the antibiotic agents prescribed in long-term as well as acute care hospitals. The most common drugs administered inappropriately were FQ and 3G CEP, which accounted for $48.2 \%$ of all inappropriately prescribed antibiotics (Additional file 3: Supplement 11). Considering the limitations of the workforce and infrastructure, implementing ASP focusing on drugs most frequently used, should be considered for non-teaching community hospitals.

The proportion of inappropriate antibiotic prescriptions exceeded $30 \%$ in this study, and was higher than the result $(26.1 \%)$ of a previous study conducted in 75 hospitals - the majority of which were large academic hospitals-in South Korea [21]. Of the inappropriate antibiotic prescriptions for the treatment of infectious diseases, a substantial proportion seemed to be caused by inappropriate diagnosis. Indeed, the proportion of appropriate 
Table 5 Appropriateness of antibiotic prescriptions in infectious cases

\begin{tabular}{|c|c|c|c|c|}
\hline & Long-term care hospitals & Acute care hospitals & $P$-value & All hospitals \\
\hline \multicolumn{5}{|l|}{ Appropriateness of diagnosis for infectious diseases ${ }^{1}(\%)$} \\
\hline By each antibiotic & $282 / 370(76.2)$ & $119 / 155(76.8)$ & 0.078 & $401 / 525(76.4)$ \\
\hline By each patient & $191 / 260(73.5)$ & 93/123 (75.6) & 0.122 & $284 / 383(74.2)$ \\
\hline Appropriateness of antibiotic prescription, by each antibiotic (\%) & & & $<0.001$ & \\
\hline Optimal & $49 / 370(13.2)$ & $65 / 155(41.9)$ & & $114 / 525(21.7)$ \\
\hline Suboptimal & $29 / 370(7.8)$ & $12 / 155(7.7)$ & & $41 / 525(7.8)$ \\
\hline Inappropriate & $98 / 370(26.5)$ & $54 / 155(34.8)$ & & $152 / 525(29.0)$ \\
\hline $\mathrm{N} / \mathrm{A}$ & 194/370 (52.4) & 24/155 (15.5) & & $218 / 525(41.5)$ \\
\hline Appropriateness of antibiotic, by each patient (\%) & & & $<0.001$ & \\
\hline Optimal & 29/260 (11.2) & 48/123 (39.0) & & $77 / 383(20.1)$ \\
\hline Suboptimal: one or more antibiotics were suboptimal & $26 / 260(10.0)$ & $9 / 123(7.3)$ & & $35 / 383(9.1)$ \\
\hline Suboptimal: unnecessary combination therapy & $1 / 260(0.4)$ & $3 / 123(2.4)$ & & $4 / 383(1.0)$ \\
\hline Inappropriate & $75 / 260(28.8)$ & $41 / 123(33.3)$ & & $116 / 383(30.3)$ \\
\hline N/A & $129 / 260(49.6)$ & 22/123 (17.9) & & 151/383 (39.4) \\
\hline $\begin{array}{l}\text { Appropriateness of antibiotic prescription only in cases with the } \\
\text { appropriate diagnosis, by each antibiotic (\%) }\end{array}$ & & & $<0.001$ & \\
\hline Optimal & 49/282 (17.4) & $64 / 119(53.8)$ & & $113 / 401(28.2)$ \\
\hline Suboptimal & 27/282 (9.6) & $9 / 119(7.6)$ & & $36 / 401(9.0)$ \\
\hline Inappropriate & $53 / 282(18.8)$ & $32 / 119(26.9)$ & & $85 / 401(21.2)$ \\
\hline $\mathrm{N} / \mathrm{A}$ & $153 / 282(54.3)$ & $14 / 119(11.8)$ & & 167/401 (41.6) \\
\hline $\begin{array}{l}\text { Appropriateness of antibiotic prescription only in cases with the } \\
\text { appropriate diagnosis, by each patient (\%) }\end{array}$ & & & $<0.001$ & \\
\hline Optimal & 29/191 (15.2) & 48/93 (51.6) & & $77 / 284(27.1)$ \\
\hline Suboptimal: one or more antibiotics were suboptimal & 24/191 (12.6) & $7 / 93(7.5)$ & & $31 / 284(10.9)$ \\
\hline Suboptimal: unnecessary combination therapy & $1 / 191(0.5)$ & 3/93 (3.2) & & $4 / 284(1.4)$ \\
\hline Inappropriate & 43/191 (22.5) & 23/93 (24.7) & & $66 / 284(23.2)$ \\
\hline N/A & $94 / 191(49.2)$ & $12 / 93(12.9)$ & & $106 / 284(37.3)$ \\
\hline
\end{tabular}

${ }^{1}$ In the cases when antibiotics were prescribed for the treatment of infectious diseases

antibiotic prescriptions increased when cases with inappropriate diagnoses were excluded from the study. Previous studies have indicated that uncertainty of diagnosis was associated with an increase in antibiotic prescriptions, and that a large amount of inappropriate antibiotic prescriptions was attributable to deficits in diagnosis, rather than therapeutic knowledge [22, 23]. These results suggest that to improve antibiotic usage patterns, interventions focusing only on the judicious use of antibiotics are likely to be insufficient; efforts to enhance physicians' diagnostic abilities will also be necessary.

Cases without sufficient data for the evaluation of the appropriateness were more often found in long-term care hospitals than acute care hospitals. Most of these cases did not have the results of creatinine levels, which is needed to adjust the antibiotic dose. This result might be associated with differences in the reimbursement system of national health insurance: diagnosis-related group reimbursements are mainly applied to long-term care hospitals, whereas fee-for-service reimbursement is primarily applied to acute care hospitals. Therefore, minimizing tests might have a positive effect for revenue in long-term care hospitals. This could also explain the phenomenon of not actively performing culture tests for identifying causative pathogens in long-term care hospitals. Therefore, to increase the appropriateness of antibiotic prescriptions, even in long-term care hospitals in South Korea, providing adequate reimbursement for essential tests to improve antibiotic use is necessary.

Our data demonstrated that the proportion of inappropriate antibiotic prescriptions was more common in prophylactic use for surgical site infection, than in therapeutic use for the treatment of infectious diseases. Prolonged antibiotic use after an operation was the major reason for 'inappropriate' antibiotic use (data not shown). In South Korea, the Health Insurance Review and Assessment Service, as part of the National Quality Assessment Program, has been assessing surgical prophylactic antibiotics in each hospital since 2007, to decrease the abuse and misuse of antibiotics [24]. As a result of this program, significant improvements have been reported in the administration of prophylactic antibiotics within 
one hour of surgical incision, and in the reduction in the use of inappropriate antibiotics, such as 3G CEP or aminoglycosides [24]. Since the inappropriate duration of antibiotic use was newly introduced in 2020, as a measurement of the program [17], the prolonged use of surgical prophylactic antibiotics might reduce in the near future.

Additionally, there was not enough workforce to ensure the successful implementation of ASP in the participant hospitals: there was a limited number of infectious disease specialists (IDS) (only one hospital employed IDS, data not shown), as well as pharmacists and staff for information technology (Table 1). Given that the amount of antibiotic use in non-teaching community hospitals is similar to those in large hospitals, investment to increase the staffing for such a workforce is necessary $[18,19]$. IDS play a major role in managing patients with various infectious diseases, and in the organization of ASP in each hospital $[25,26]$. However, despite the importance of their role, the number of IDS in South Korea remains insufficient; therefore, it is difficult to find them in nonteaching community hospitals. In 2020, there were only 242 IDS, most of whom were employed in large hospitals [27, 28]. Given the shortage of workforce, less labour-intensive ASP strategies, such as syndrome-based intervention against common infectious diseases, may be a potential solution to improve the appropriateness of antibiotic use in non-teaching community hospitals [29]. Furthermore, a collaborative, consultation network focused on ASP implementation in non-teaching community hospitals, and supported by large hospitals with sufficient infrastructure for ASP, is necessary in South Korea [30]. Fortunately, the recently announced Korean National Action Plan on Antimicrobial Resistance 20212025 includes a plan to conduct a pilot project about the consultation network of ASP as a countermeasure for antimicrobial resistance in non-teaching community hospitals [31].

There are several potential limitations in the present study. First, we did not collect data on patients' clinical information. Therefore, the appropriateness of antibiotic use could not be determined in some cases. Second, a large proportion of antibiotics and patients could not be fully evaluated because of insufficient data. To overcome this shortcoming, the appropriateness of antibiotics was presented in the subdivision as well: route of administration, dose, and antibiotic choice. Third, the generalizability of our results is limited because the characteristics of the nine hospitals may not be representative of all nonteaching community hospitals in South Korea. Indeed, there are more than 3000 non-teaching community hospitals in South Korea. Additionally, selection bias might exist because we recruited all hospitals that voluntarily submitted applications for participation. Considering the healthcare system in South Korea, which is well controlled by National Health Insurance, and considering the limited workforce for ASP in primary care or long-term care hospitals, the situation might not be different in other non-teaching community hospitals [27]. Finally, the study did not properly evaluate the proportion of patients receiving antibiotics from all inpatients during the study period, which would better reflect the burden of antibiotic use.

\section{Conclusion}

The antibiotic usage patterns vary among non-teaching community hospitals in South Korea. The proportion of inappropriate antibiotic prescriptions exceeds $30 \%$ of the total antibiotic prescriptions, and the inaccurate diagnosis seems to be positively associated with inappropriate antibiotic prescriptions. Given the limited workforce and infrastructure, customized strategies for these hospitals and national-level support are necessary to improve ASP in non-teaching community hospitals in South Korea.

\section{Abbreviations}

ASP: Antimicrobial stewardship program; DDD: Defined daily dose; DOT: Days of therapy; 1 G CEP: First generation cephalosporin; 2G CEP: Second generation cephalosporin; 3 G CEP: Third generation cephalosporin; BL/BLI: $\beta$-Lactam/ $\beta$ lactamase inhibitor; FQ: Fluoroquinolone; IDS: Infectious disease specialists.

\section{Supplementary Information}

The online version contains supplementary material available at https://doi. org/10.1186/s13756-022-01082-2.

Additional file 1: Supplement 1. Data collection form (for investigators in each hospital).

Additional file 2: Supplement 2. Evaluation of appropriateness of antibiotic prescription (for infectious diseases specialists).

Additional file 3: Supplement 3-12.

\section{Acknowledgements}

We would like to acknowledge all the hospitals in this study for their participation in the survey. The authors are also grateful to Jinwoo Yang (Onnuri Convalescent Hospital), Hyunok Park (Cheongju Hana General Hospital), Ji Hoon Kang (Daejeon Dongbu Convalescent Hospital), Myoungsuk Yun (Incheon Eun-Hye Hospital), and Young Taek Kim/Hye Jung Lee (Cheil Orthopedic Hospital) for their assistance with data collection. We would also like to thank Woochang Hwang from the Biostatistical Consulting and Research Lab, Medical Research Collaborating Center, Industry-University Cooperation Foundation, Hanyang University for statistical advice.

\section{Authors' contributions}

Conceptualization: B.K; Methodology: Y.C.K., J.Y.P., B.K., E.S.K., H.G., R.M., S.Y.P., M.J.L., S.M.M., S.H.P., K.H.S., H.B.K.; Software: R.M. and J.Y.P.; Validation: Y.C.K., J.Y.P., B.K., S.Y.P., M.J.L., and S.M.M.; Formal analysis: R.M. and J.Y.P.; Investigation:Y.C.K., J.Y.P., B.K., E.S.K., H.G., R.M., S.Y.P., M.J.L., S.M.M., S.H.P., K.H.S., H.B.K.; Resources: Y.C.K., J.Y.P., B.K., E.S.K., H.G., R.M., S.Y.P., M.J.L., S.M.M., S.H.P., K.H.S., H.B.K.; Data curation:Y.C.K., J.Y.P., B.K., S.Y.P., M.J.L., and S.M.M; Writing-original draft:Y.C.K., J.Y.P..; Writing - review and editing: B.K. and E.S.K.; Visualization-R.M. and J.Y.P.; 
Supervision: E.S.K., H.B.K.; Project administration: B.K.; Funding acquisition: B.K. All authors read and approved the final manuscript.

\section{Funding}

This work was supported by the Research Program funded by the Korea Disease Control and Prevention Agency (Grant Number 20200909F55-00), a grant from the Bio \& Medical Technology Development Program of the National Research Foundation (NRF) and funded by the Korean government (MSIT) (Funding No. 2019M3E5D1A01066063). The funders had no role in the study design, data collection, analysis, preparation of the manuscript, or the decision to publish.

\section{Availability of data and materials}

The datasets used and/or analyzed during the current study are available from the corresponding author on reasonable request.

\section{Declarations}

\section{Ethics approval and consent to participate}

The study's protocol was approved by the Institutional Review Board of Hanyang University, Seoul Hospital (IRB no. 2020-12-040). The requirement for an informed written consent from patients was waived because of the retrospective nature of the study.

\section{Consent for publication}

Not applicable.

\section{Competing interests}

The authors declare that they have no competing interests.

\begin{abstract}
Author details
'Department of Internal Medicine, Division of Infectious Diseases, Yongin Severance Hospital, Yonsei University College of Medicine, Yongin, Korea. ${ }^{2}$ Department of Pediatrics, Chung-Ang University Hospital, Seoul, Korea. ${ }^{3}$ Department of Internal Medicine, Hanyang University College of Medicine, 222-1, Wangsimni-ro, Seongdong-gu, Seoul 04763, Korea. ${ }^{4}$ Division of Infectious Diseases, Department of Internal Medicine, Seoul National University Bundang Hospital, Seoul National University College of Medicine, 82 Gumi-ro, 173 beon-gil, Bundang-gu, Seongnam 13620, Gyeonggi-do, Korea. ${ }^{5}$ Incheon Eun-Hye Hospital, Incheon, Korea. ${ }^{6}$ Department of Economics, College of Political Science and Economics, Korea University, Seoul, Korea. ${ }^{7}$ Division of Infectious Diseases, Department of Internal Medicine, Soonchunhyang University Seoul Hospital, Soonchunhyang University College of Medicine, Seoul, Korea. ${ }^{8}$ Department of Internal Medicine, Inje University Sanggye-Paik Hospital, Seoul, Korea. ${ }^{9}$ Division of Infectious Diseases, Department of Internal Medicine, Hallym University Sacred Heart Hospital, Anyang, Korea. ${ }^{10}$ Division of Infectious Diseases, Department of Internal Medicine, College of Medicine The Catholic University of Korea, Seoul, Korea.
\end{abstract}

\section{Received: 14 December 2021 Accepted: 12 February 2022}

Published online: 22 February 2022

\section{References}

1. Gaieski DF, Mikkelsen ME, Band RA, Pines JM, Massone R, Furia FF, Shofer FS, Goyal M. Impact of time to antibiotics on survival in patients with severe sepsis or septic shock in whom early goal-directed therapy was initiated in the emergency department* Crit Care Med. 2010;38(4):1045-53.

2. Liu VX, Fielding-Singh V, Greene JD, Baker JM, Iwashyna TJ, Bhattacharya J, Escobar GJ. The timing of early antibiotics and hospital mortality in sepsis. Am J Respir Crit Care Med. 2017;196(7):856-63.

3. Combating Antimicrobial Resistance: Policy Recommendations to Save Lives. Clinical Infectious Diseases 2011, 52(suppl_5):S397-S428.

4. The Review on Antimicrobial Resistance. Tackling drug-resistant infections globally: final report and recommendations. 2016. Available at: https://amr-review.org/sites/default/files/160518_Final\%20paper_with\% 20cover.pdf. Accessed 19 Nov 2021.
5. Srigley JA, Brooks A, Sung M, Yamamura D, Haider S, Mertz D. Inappropriate use of antibiotics and Clostridium difficile infection. Am J Infect Control. 2013:41(11):1116-8.

6. Jourdan A, Sangha B, Kim E, Nawaz S, Malik V, Vij R, Sekhsaria S. Antibiotic hypersensitivity and adverse reactions: management and implications in clinical practice. Allergy Asthma Clin Immunol. 2020;16(1):6.

7. Pollack LA, Srinivasan A. Core elements of hospital antibiotic stewardship programs from the centers for disease control and prevention. Clin Infect Dis 2014, 59(suppl_3):S97-S100.

8. Stenehjem E, Hyun DY, Septimus E, Yu KC, Meyer M, Raj D, Srinivasan A. Antibiotic stewardship in small hospitals: barriers and potential solutions. Clin Infect Dis. 2017;65(4):691-6.

9. Ibrahim OM, Polk RE. Benchmarking antimicrobial drug use in hospitals. Expert Rev Anti Infect Ther. 2014;10(4):445-57.

10. Kim B, Hwang H, Kim J, Lee M-j, Pai H: Ten-year trends in antibiotic usage at a tertiary care hospital in Korea, 2004 to 2013. Korean J Internal Med. 2020; 35(3):703-13.

11. Kim YC, Kim MH, Song JE, Ahn JY, Oh DH, Kweon OM, Lee D, Kim SB, Kim $\mathrm{H}-\mathrm{W}$, Jeong SJ et al. Trend of methicillin-resistant Staphylococcus aureus (MRSA) bacteremia in an institution with a high rate of MRSA after the reinforcement of antibiotic stewardship and hand hygiene. Am J Infect Control 2013, 41(5):e39-e43.

12. Ryu S. The new Korean action plan for containment of antimicrobial resistance. J Glob Antimicrobial Resist. 2017;8:70-3.

13. Health Insurance Review and Assessment Service. Healthcare Bigdata Hub. Available from: https://opendata.hira.or.kr/home.do. Accessed 1 Nov 2021.

14. World Health Organization (WHO). ATC/DDD index: updates included in the ATC/DDD index. 2021. Available at: https://www.whocc.no/atc ddd_index/updates_included_in_the_atc_ddd_index/. Accessed 13 Nov 2021.

15. Gyssens IC. Quality measures of antimicrobial drug use. Int J Antimicrob Agents. 2001;17(1):9-19.

16. Gilbert DN, Chambers HF, Eliopoulos GM, Saag MS, Pavia AT. The Sanford guide to antimicrobial therapy. 49th ed. 2019.

17. Health Insurance Review and Assessment Service. The 9th nationwide evaluation of the appropriateness of surgical prophylactic antibiotics in Korean hospitals in 2020. Available at: http://www.hira.or.kr/bbsDummy. do?pgmid $=$ HIRAA020002000100\&brdScnBltNo $=4 \& b r d B l t \mathrm{No}=7623 \#$ none/. Assessed 12 Dec 2020

18. Stenehjem E, Hersh AL, Sheng X, Jones P, Buckel WR, Lloyd JF, Howe S, Evans RS, Greene T, Pavia AT. Antibiotic use in small community hospitals. Clin Infect Dis. 2016;63(10):1273-80.

19. Magill SS, Edwards JR, Beldavs ZG, Dumyati G, Janelle SJ, Kainer MA, Lynfield R, Nadle J, Neuhauser MM, Ray SM, et al. Prevalence of antimicrobial use in US Acute Care Hospitals, May-September 2011. JAMA. 2014;312(14):1438-46.

20. Kim B, Kim Y, Hwang H, Kim J, Kim S-W, Bae I-G, Choi WS, Jung SI, Jeong $\mathrm{HW}$, Pai $\mathrm{H}$ : Trends and correlation between antibiotic usage and resistance pattern among hospitalized patients at university hospitals in Korea, 2004 to 2012. Medicine 2018;97(51):e13719.

21. Moon SM, Kim B, Park SY, Lee MJ, Park JY, Jung J, Park JS, Song K-H, Chang $\mathrm{H}-\mathrm{H}$, Song J-Y et al. Point prevalence survey for appropriateness of antibiotic prescription in 75 Korean hospitals. 2021 Spring Conference of Korean Society for Antimicrobial Therapy.

22. Wang D, Liu C, Zhang X, Liu C. Does diagnostic uncertainty increase antibiotic prescribing in primary care? NPJ Primary Care Respir Med. 2021:31(1):17

23. Xue H, Shi Y, Huang L, Yi H, Zhou H, Zhou C, Kotb S, Tucker JD. Sylvia SY: C. J Antimicrobial Chemother. 2019;74(1):256-63.

24. Kim ES, Park S-W, Lee C-S, Gyung Kwak Y, Moon C, Kim B-N. Impact of a national hospital evaluation program using clinical performance indicators on the use of surgical antibiotic prophylaxis in Korea. Int J Infect Dis. 2012;16(3):e187-92.

25. Ragnar Norrby S. Infectious disease emergencies: role of the infectious disease specialist. Clin Microbiol Infect. 2005;11:9-11.

26. Ostrowsky B, Banerjee R, Bonomo RA, Cosgrove SE, Davidson L, Doron S, Gilbert DN, Jezek A, Lynch JB, Septimus EJ, et al. Infectious diseases physicians: leading the way in antimicrobial stewardship. Clin Infect Dis. 2018;66(7):995-1003. 
27. Lee MJ, Moon SM, Kim B, Park SY, Park JY, Koo H, Lee H, Song K-H, Lee H, Park JS, et al. Status of antimicrobial stewardship programmes in Korean hospitals including small to medium-sized hospitals and the awareness and demands of physicians: a nationwide survey in 2020. J Glob Antimicrobial Resist. 2021;26:180-7.

28. Jang Y, Park SY, Kim B, Lee E, Lee S, Son H-J, Park JW, Yu SN, Kim T, Jeon $\mathrm{MH}$, et al. Infectious diseases physician workforce in Korea. J Korean Med Sci. 2020;35(49):e428.

29. Mediwala KN, Kohn JE, Bookstaver PB, Justo JA, Rac H, Tucker K, Lashkova L, Dash S, Al-Hasan MN. Syndrome-specific versus prospective audit and feedback interventions for reducing use of broad-spectrum antimicrobial agents. Am J Infect Control. 2019;47(11):1284-9.

30. Moehring RW, Yarrington ME, Davis AE, Dyer AP, Johnson MD, Jones TM, Spires SS, Anderson DJ, Sexton DJ, Dodds Ashley ES. Effects of a collaborative, community hospital network for antimicrobial stewardship program implementation. Clin Infect Dis. 2021;73(9):1656-63.

31. Ministry of Health and Welfare of Korea. The Korean National Action Plan on antimicrobial resistance 2021-2025. Available at: http://www.mohw. go.kr/react/al/sal0301vw.jsp?PAR_MENU_ID=04\&MENU_ID=0403\& page $=1 \&$ CONT_SEQ=368388. Accessed 5 Feb 2022.

\section{Publisher's Note}

Springer Nature remains neutral with regard to jurisdictional claims in published maps and institutional affiliations.

- fast, convenient online submission

- thorough peer review by experienced researchers in your field

- rapid publication on acceptance

- support for research data, including large and complex data types

- gold Open Access which fosters wider collaboration and increased citations

- maximum visibility for your research: over 100M website views per year

At BMC, research is always in progress.

Learn more biomedcentral.com/submissions 